\title{
POLÉMICA Y OPORTUNIDAD: LA FUNCIÓN DE LAS MINORÍAS JUDÍAS EN EL PENSAMIENTO DE GREGORIO DE ELVIRA
}

\author{
POR \\ José Antonio Molina Gómez ${ }^{1}$ \\ Universidad de Murcia
}

RESUMEN

Las principales obras de Gregorio de Elvira son comentarios bíblicos donde el autor refleja cuestiones teológicas y doctrinales de gran importancia al final del siglo IV, pero también percibimos a través de sus obras concepciones de poder y sobre la sociedad, sobre todo, el conflicto entre la Iglesia (plebs sancta) y los judíos (plebs praua). Su testimonio es uno de las fuentes históricas más importantes en la Hispania cristiana de la Antigüedad tardía.

PALABRAS CLAVE: Gregorio de Elvira; siglo IV; Antigüedad tardía; España tardorromana; comentario bíblico.

\section{POLEMIC AND OPPORTUNITY: THE ROLE OF JEWISH MINORITIES IN THE THOUGHT OF GREGORY OF ELVIRA}

\begin{abstract}
Gregory of Elvira main works are biblical commentaries where he reflects doctrinal and theological questions of great meaning at the end of the 4th century, but also we realize through his pages conceptions of power and about society, above all, the conflict between the Church (plebs sancta) and the jews (plebs praua). His testi-mony is one of the most important historical sources in Christian Hispania during the Late Antiquity.
\end{abstract}

KEY WORDS: Gregory of Elvira; 4th Century; Late Roman Empire; Late Roman Spain; Biblical commentary.

Cómo CITAR ESTE ARTículo / CITATION: Molina Gómez, José Antonio. 2021. «Polémica y oportunidad: la función de las minorías judías en el pensamiento de Gregorio de Elvira». Hispania Sacra LXXIII, 147: 61-66. https://doi.org/10.3989/hs.2021.005

Recibido/Received 21-02-2019

Aceptado/Accepted 13-01-2020

\section{INTRODUCCIÓN}

Gregorio de Elvira (muerto hacia 392) concibe la Iglesia como centro y eje de la vida cristiana. ${ }^{2}$ Sin embargo, no puede dejar de ver que fuera de los límites de la comunidad existe un entorno aparentemente periférico y situado de momento al margen de los planes de salvación universal, formado por una amalgama de judíos, paganos y herejes. Vamos a abordar a continuación la comprensión de Gregorio de Elvira con respecto a su percepción de las relaciones

\footnotetext{
1 jamolgom@um.es /
}

ORCID iD: https://orcid.org/0000-0003-4675-0125

2 Mazorra 1947; Buckley 1964; Codoñer Merino 1982; Domínguez del Val 1973; Molina 2000, 20-43 (con bibliografía sobre la personalidad literaria del Gregorio de Elvira y su pensamiento); Rodríguez Ratia 2017. con los judíos (visible principalmente en sus Tractatus pero también en otros pasajes de su obra). ${ }^{3}$ A través de un lenguaje eminentemente teológico y desde el género homilético, Gregorio de Elvira se adentra en la concepción de Ecclesia como comunidad religiosa, social y política. En los límites exteriores de dicha comunidad se concentran una variedad de paganos (aparentemente residual), herejes y judíos que conforman la marginalidad simbólica en la Hispania de la segunda mitad del siglo IV. El debate es eminentemente erudito, teórico y exegético, si bien en el caso de la polémica antijudía se registran no pocos elementos vivenciales (usos, costumbres, rituales); teniendo en cuenta que la presencia de una comunidad judía en la Elvira romana

\footnotetext{
3 Molina Gómez 2000, 183-194.
} 
está atestiguada, ${ }^{4}$ las menciones que hace su obispo hacia este colectivo merecen ser examinadas con un detalle especial pues la polémica transciende el ámbito meramente teórico para una época en la que no abundan precisamente las fuentes documentales. Las alusiones al paganismo en la obra gregoriana son escasas, el verdadero centro de su polémica y en torno al cual se materializa su visión de la comunidad es, en el caso que nos ocupa, el gran debate contra la Sinagoga.

\section{EL JUDAÍSMO EN LA OBRA DE GREGORIO DE ELVIRA}

\section{La Sinagoga y la Iglesia}

Gregorio de Elvira se refiere abundantemente a los judíos como es habitual en la patrística de la época. ${ }^{5}$ Los judíos son un pueblo (natio, plebs) que había convivido largo tiempo en el seno del Imperio romano con paganos y cristianos. Aunque en el Bajo Imperio la cultura se va haciendo progresivamente más cristiana y el credo niceno llegará a adquirir fuerza de ley no sin esfuerzo en medio de graves conflictos religiosos, siguen conviviendo a mediados del siglo IV cristianos (tanto "ortodoxos» como "heterodoxos»), con judíos observadores de sus ritos (circuncisión y sabath), y «gentiles» fieles a la que había sido legalmente hasta entonces la religión nacional romana. Gregorio de Elvira convive, además literalmente, ${ }^{6}$ con estas "alternativas» religiosas y es precisamente a través de su polémica antijudaizante donde percibimos por contraste y autoafirmación la construcción social de la identidad cristiana, la formulación de su propio ideal donde se prefigura una noción sacralizada de la comunidad, una plebs sancta. Sirviéndose del método alegórico ${ }^{7}$ en la interpretación de las Sagradas Escrituras, formula no tanto una crítica a la periferia hostil del cristianismo (que ya había empezado a quedar definido en el célebre concilio de Elvira que habría tenido lugar algunos años antes en la sede del propio San Gregorio), ${ }^{8}$ como un ejercicio de autodefinición y de permanencia no solo en el credo niceno, sino en la tradición apostólica en la que el pueblo cristiano es siempre una apostolica plebs. ${ }^{9}$

\section{Lex prisca y coherencia en la historia de la salvación}

La «cuestión judía» en Gregorio de Elvira es primero exegética porque reivindica como patrimonio para la causa cristiana enteramente el Antiguo Testamento. Su visión no puede ser, por tanto, más programática. La polémica antijudía le sirve para definir aquello que le preocupa, que no es la exclusión política de los judíos, no estamos en ese momento aún, sino la definición, la autocomprensión de la propia comunidad cristiana, su posición en la historia y su destino soteriológico final que no solo la emancipa de la Sinagoga,

Molina Gómez 2000, 183-193.

Blumenkranz 1960, 215; Taylor 1961.

Thouvenot 1943; Lomas 1994; Sayas Abengoechea 1993; García Iglesias 1978; Suárez Fernández 1980; García Moreno 1993.

7 Molina Prieto 1998.

8 Molina Gómez 2000, 48-57; a la bibliografía allí citada sobre un tema que jamás perderá interés se pueden añadir Barcala Muñoz 2003, 161-192 y Teja 2005, 197-228; Molina Gómez y Mateo Donet 2018.

9 Fernández Ardanaz 1983. sino que consagra a la Ecclesia $^{10}$ como su sucesora y heredera natural. Gregorio no pretende dar ningún dato objetivo, no es aquí un "testigo» de un certamen aduersos ludadeos, que nos dé información positiva de cuántos judíos vivían, y cómo, en la lliberri cristiana. La predicación antijudía es sobre todo una oportunidad de debate y de presentación de las propias concepciones al pueblo fiel, pueblo que será el "verdadero» Israel a partir de ese momento de acuerdo con la nueva filosofía de la historia imperante. Debemos entenderlo no desde la perspectiva de una fuente objetiva sino desde la construcción de la alteridad.

Gregorio de Elvira identificaba la ley judía con el pasado venerable, pero superado; la ley judía era la lex prisca, identificada con la tradición judía del Antiguo Testamento y que desde el punto de vista cristiano debía ser considerada como derogada. De acuerdo con las concepciones históricas de la nueva filosofía de la historia, la culminación de los tiempos había llegado con Cristo, cerrando el ciclo de la Sinagoga y abriendo la historia universal de la salvación.

Los cristianos habrían de ser considerados en lo sucesivo como los verdaderos hebreos, los continuadores auténticos de Abraham y Moisés, quienes en virtud de la exégesis tipológica tan del gusto de Gregorio de Elvira, ya habrían llevado una vida verdaderamente cristiana. ${ }^{11}$ La esencia de lo cristiano se respiraría en ambos Testamentos; perseverar solo en la ley antigua, en los usos y ritos judíos, era concebido como un error histórico que relegaba a la Sinagoga a la categoría de pueblo díscolo, apartado, por ahora, del curso general de la historia de la salvación. ${ }^{12} \mathrm{Y}$ así no estamos solo ante la sustitución de un pueblo elegido (el de la Sinagoga) por otro (el de la Ecclesia), cuanto más ante la sanción de un hecho antiguo y entendido como cierto: que el mensaje cristiano era ya una realidad desde los lejanos tiempos de los patriarcas. La conversión del pueblo de la Sinagoga es algo esperado con ansia; representará, no solo la ocasión para la reconciliación (ambos pueblos tienen en Abraham al padre común) sino la materialización de la victoria final de Cristo y su pueblo. Así se expresa la historia como providencia universal. En este sentido, dicha «reconciliación» solo es tal ante los ojos de la del predicador, pues se está llegando a la creación de un sistema cada vez más coercitivo en el que la Iglesia se involucra con el aparato estatal para crear una respublica Christiana. ${ }^{13}$

Para Gregorio de Elvira no hay ninguna duda de que la reconciliación entre la Sinagoga y la Iglesia supondrá el nacimiento de un pueblo nuevo, lo cual supondrá la culminación de los tiempos históricos. Israel tiene un destino glorioso al lado de los cristianos, una vez ambos pueblos se hayan reconciliado. La integración del pueblo de la Sinagoga en el discurso gregoriano se hace obsesivamente en el seno de la interpretación bíblica. La interpretación recurre a pasajes del Antiguo Testamento que se entienden cristológicamente y desde la exégesis unificadora que convierte en una unidad los dos Testamentos, siguiendo un esquema finalista y mesiánico. ${ }^{14}$ Puede decirse que se basa en la apropiación de la tradición judaica al servicio de una eclesiología fuertemente

10 Collantes 1954.

11 Simonetti 1985b.

12 Quasten 1941, 481-487.

13 Molina Gómez 2000, 176.

14 Ladaria 1999. 
militante que va a presentarse en breve como una sacra respublica. La polémica antijudía es programática, intelectualmente centrada en el texto escrito, de coherencia hermenéutica. ${ }^{15}$ Por oposición al ritual judío los nuevos misterios de la predicación cristiana consideran derogada la vigencia histórica de la prisca lex ante la culminación del hecho mesiánico. ${ }^{16}$

Gregorio de Elvira se sirve de una metodología exegética de carácter tipológico, en virtud de la cual se encuentra un sentido profundo en la Escritura que la tradición judía persistiría, a su juicio, en ignorar. ${ }^{17}$ Desde este punto de vista, y de manera significativa, en la Biblia se revela tanto el destino del pueblo de la Sinagoga como el de la Ecclesia, pues la coherencia y veracidad de ambos Testamentos demuestra que la tradición hebrea está inacabada solo si se entiende el Antiguo Testamento como prefiguración del Nuevo. Para ello, poniéndose aparentemente a disposición del texto, pero en realidad proyectando ya una solución, el obispo pretende, como guardián social de la sabiduría, afrontar el estudio de las etimologías del hebreo, ${ }^{18}$ para extraer el verdadero sentido de estas, que habría sido ignorado por la exégesis rabínica. El punto de unión común de ambos pueblos, judío y cristiano, es la literatura bíblica, por eso la exégesis gregoriana se centra en los textos del Antiguo Testamento, que se aborda desde formas de interpretación y exégesis que son de tradición relativamente obsoleta en tiempos del obispo. En términos modernos Gregorio de Elvira, que tantos esfuerzos de predicación y misión hace para que su comunidad advierta las directrices fundamentales de todos los tipos y figuras que explican las historias del Antiguo Testamento enlazándolas con el Nuevo, es un ideólogo con una misión: otorgar una explicación a toda la historia humana basándose en la exégesis bíblica y la contraposición a la Sinagoga.

Dentro de esta coherencia intertestamentaria los personajes de la Antigüedad judía son ya cristianos ante litteram. Por eso al hablar de las teofanías del Antiguo Testamento se afirma que ya el Hijo de Dios fue visto, y reconocido, por los patriarcas. La nueva tradición cristiana, de la que el sistema hermenéutico gregoriano es un buen ejemplo, sincroniza el Antiguo Testamento con el Nuevo y los hace contemporizar entre sí. Moisés como caudillo es el tipo de Cristo como $d u x$, pues se trata en ambos casos del mismo conductor del pueblo de Dios. No en vano es la figura principal del Tratado VII, y su trayectoria heroica como conductor del pueblo elegido es interpretada como metáfora "escenográfica», ${ }^{19}$ que explica el drama de la salvación y la liturgia bautismal. Al igual que ocurría con Abraham y José, cada uno de los personajes que lo rodean y cada uno de sus acontecimientos biográficos son en realidad prefiguraciones, que contienen en sí mismas el mensaje de la salvación. Moisés tiene un poderoso antagonista que sin embargo es vencido, el Faraón, imagen antagónica del anticristo, tanto como Egipto representa al mundo terrenal abocado a la corrupción. El pueblo

\footnotetext{
15 Dulaey 1996.

16 Simon 1948. Seaver 1952, 51. Noethlichs 1996. Rengstorf y Kortzfleisch. 1968, 84.

17 Para el método exegético empleado por Gregorio de Evira, véase Molina Gómez 2000, 73-109

18 Molina Gómez, 2007.

19 Tovar Paz 1994, 279-284.
}

de Israel prefiguraba al hombre, sumido en la servidumbre del pecado, y a quien Cristo había de salvar al final de los tiempos liberándolo del peso de la historia «Aegyptus itaque figura saeculi fuit, Pharao diaboli, filii Israel protoplasti imaginem induerant, ex cuius origine censebantur; Moyses autem, qui eos missus est liberare, typum Xpisti gerebat». ${ }^{20}$

Los hechos narrados se entienden desde una interpretación figurada, en virtud de la comparación tipológica de Moisés con Cristo que hace que todo acontecimiento responda a un plan que demuestra que el Salvador ya estaría presente en la Escritura. La exégesis gregoriana se vuelve audaz en su proceso de expropiación de la tradición judía a sus legítimos propietarios para forjar un nuevo modo de pensar. Y así, las parteras de los judíos cuando se niegan a eliminar a los varones tal y como les había ordenado el faraón, realizan realmente en ese momento su contribución al drama mesiánico y simbólicamente se independizan al mismo tiempo de la Sinagoga:

Obstetrices istae, qui non oboedierunt Pharaoni, animae sunt mentesque sanctorum, quae Pharaoni diabolo non solum non obtemperare uolebant, uerum masculos, id est uirtutes bonorum operum quasi quemdam diuini seminis partum de cordis utero obstetricantes in lucem cogitationis producebant, ut robor fidei praeualeret. $^{21}$

El verdadero protagonista de la historia sagrada es el pueblo cristiano, cuya presencia en el Antiguo Testamento se rastrea a través de las innumerables tipologías. Gregorio de Elvira se apoya con fuerza en la tradición exegética norteafricana. Así concibe la derogación de la Ley y la proclamación del triunfo de Cristo. La rica tradición polemista en torno a los ritos judaizantes le sirve para igual propósito; la circuncisión o el sabbath son signo de una antigua espiritualidad, sustituida por una creciente sacramentalización de la vida cada vez más visible en el bautismo y la penitencia.

El sentido literal e histórico de los hechos del pueblo de Israel anticipa las verdades espirituales de la fe. De esta forma, cuando se narra que la madre (tipo de la Sinagoga) de Moisés (tipo de Cristo) ocultó su nacimiento durante tres meses, Gregorio de Elvira ve ahí la alusión velada al misterio de la trinidad, elemento central en una época de grandes debates cristológicos. Su interpretación de carácter numerológico le permite entrever además la señal de acontecimientos en la vida de Cristo prefigurados en la historia de Moisés. Y así como la Sinagoga abandona a Cristo, la madre de Moisés abandona a su hijo. Gregorio de Elvira piensa que no es sino la imagen del mundo judío del cual provenía Cristo y que lo había rechazado. La interpretación tipológica que hace Gregorio sobre Moisés de nuevo en sus Tractatus le permite trazar los orígenes de este gran conflicto histórico entre Iglesia y Sinagoga: "Mater ataque Moysi figura erat synagogae patrum ac prophetarum, ex quorum origine Xpistus secundum carnem est natus»..$^{22}$

He aquí una verdadera interpretación de la historia universal desde la predicación, al mismo tiempo que señala las diferencias con la Sinagoga y marca distancias con la tradi-

20 Gregorius Ilib. Tract Orig VII 3, 17-20 (Pascual Torró 1997, 192). 196).

1 Gregorius Ilib. Tract Orig VII 11, 23-30 (Pascual Torró 1997, 
ción judía. ${ }^{23}$ Busca también en el entorno de los personajes centrales a los personajes secundarios, susceptibles de ser interpretados desde la exégesis tipológica, una exégesis que emplea para afrontar las preocupaciones de su tiempo y la necesidad de forjar la identidad cristiana y la concepción de la Ecclesia como una institución total. Las prefiguraciones de carácter profético prosiguen con los personajes que están alrededor de Moisés y Abraham. Mientras tanto, dentro de la concepción política de Gregorio de Elvira, un nuevo pueblo se une al pueblo de Dios, los antiguos gentiles reciben al mismo Cristo que la Sinagoga había rechazado; es el caso de la hija del Faraón, que recoge a Moisés de las aguas en donde lo había abandonado su propia madre. La hija del Faraón no es sino la alusión a la Iglesia; los pueblos paganos habían estado próximos al demonio, en clara alusión al culto de los ídolos como se ejemplifica a través de la hija del Faraón. Pero ese momento histórico está cerrado, la Iglesia se ha nutrido de la amalgama de pueblos que abandonaron el culto idolátrico, esa enorme diversidad étnica de gentes sustituyendo al díscolo pueblo de la Sinagoga. Con todo, la reconciliación con Israel también llegará, y con ella, la reconciliación universal y el final de la historia; por eso se sirve Gregorio de Elvira de Tertuliano para afirmar que Israel "creerá al final de los tiempos»: in nouissimis temporibus ... creditura est. ${ }^{24}$ Gregorio de Elvira será repetido e imitado en la Antigüedad cristiana así como en la Edad Media hispana, que consagrará así su visión de los judíos. ${ }^{25}$

\section{CONCLUSIONES: DESTINO HISTÓRICO Y COMUNIDAD}

La época de Gregorio de Elvira es testigo del aumento de la preeminencia sapiencial del obispo, ${ }^{26}$ que crece al mismo tiempo que aumentan sus competencias temporales, de manera que sabiduría y poder se mixtifican. El obispo se convierte en guía, maestro, intérprete y árbitro, aunque eso no quiere decir que siempre hubiera claridad de criterios o unanimidad. ${ }^{27}$ En la homilética gregoriana existen dos conflictos abiertos. Por un lado el problema de las herejías y por otro el trato con el judaísmo. El obispo se erigió desde el punto de vista sapiencial, y de manera gradual también desde el punto de vista temporal, en nuevo maestro y guía tanto civil como sapiencial, que podía clamar de manera categórica contra el hereje. ${ }^{28}$ Aunque la progresiva mezcla de la Iglesia en asuntos temporales y políticos propiciara situaciones de jerarquización desde donde resultara más fácil lanzar un anatema de carácter vinculante y coercitivo, el problema de las heterodoxias es entendido, por los propios Padres, no tanto un problema político como un acto de disensión contra la humanidad. El criterio para definir la exégesis correcta se apoyaba considerablemente y desde finales del siglo IV en la fidelidad a la interpretación nicena de las Sagradas Escrituras, fidelidad que en Gregorio de Elvira pretende ser manifiesta y militante. «Nicaeni autem synodi tractatum omni animi nisu ex tota fide seruantes,

\footnotetext{
23 Montserrat Torrents 1989, 38. Taylor 1961.

24 Gregorius Ilib. Tract Orig VII 19, 25-26 (Pascual Torró 1997, 200).

25 Lejay 1908, 435-437.

26 Acerbi, Marcos y Torres 2016.

27 Young 1997, 17.

28 Gregorius Ilib. De Fide 8 (93), 14-15 (Pascual Torró 1998, 140).
}

amplectimur: hunc enim tractatum scimus contra omnes haereses inuicta ueritate oppositum $» .^{29}$ En efecto, a primera vista todo parece indicar que se trata, al menos en parte, de un problema de exégesis y de autoridad; para Gregorio de Elvira, los errores surgen por la condenable ligereza en la interpretación de las Escrituras, audacia punienda, ${ }^{30}$ pero asimismo surgen del desconocimiento, de la ignorantia, consecuencia de la falta de preparación y por el hecho de no tomarse verdaderamente en serio la labor de apostolado y cura de almas. Ya que multi sunt ineruditi homines, como dice en el Tractatus I, a los que les gusta tomar la palabra hasta retorcerla según el antiguo uso del sofista pagano, el modelo de academicus disputator, su oponente imaginario, la ficta persona de la retórica, del cual tanto abomina el predicador iliberritano en el De Fide. Ofrece como reverso, la simplicidad de la paideia cristiana, frente a las enrevesadas razones de los Ilamados peyorativamente grammatici, ${ }^{31}$ malos cristianos paganizantes, por eso mismo califica Gregorio de Elvira a los herejes de discipuli philosophorum. ${ }^{32}$ En una progresiva desconfianza ante la razón, el predicador se muestra preocupado por las argumentaciones retorcidas y alambicadas, que, bajo una apariencia de certeza, conducen al error (y de ahí a la impiedad), convirtiendo la fe en una mera cuestión de argumentación y contra-argumentación, en un debate académico cualquiera, como los de la filosofía pagana. Esta unión entre sapiencia y autoridad para el caso de las herejías la observamos también en el extremo concerniente a la relación con el judaísmo. Mientras que en De Fide encontramos ya la definición de una ortodoxia combativa y militante que propone el castigo y la exclusión de las herejías, singularmente el subordinacionsimo arriano (aunque se nombren muchas otras), el gran temor del predicador de Elvira lo constituye asimismo ejercer el control sobre la autoridad interpretativa de los pasajes del Antiguo Testamento, de ahí la intensidad de la polémica no solo con las herejías sino con el judaísmo. ${ }^{33}$ Esa es su gran preocupación, sobre todo a la hora de diferenciarse de los ritos judaizantes y de la tradición del Antiguo Testamento. Nuestro obispo asocia, pues, la herejía con el sofisma, y el error doctrinal con la mala exégesis y al judaísmo con la incompetencia en la interpretación intertestamentaria. En los Tractatus encontramos una verdadera teoría de la historia, que vemos formulada en el pensamiento exegético de Gregorio de Elvira a través de la polémica con el judaísmo. Los viejos ritos del judaísmo se deben sustituir. El bautismo hará entrar bajo la disciplina evangélica a los judíos y gentiles, quienes en un nuevo renacer entrarán a formar parte de la plebs sancta, como vemos a lo largo del Tractatus VII, por la regeneración activa del bautismo. Lo que parece una constante preocupación catequética convierte a Gregorio de Elvira ante todo en un pedagogo esforzado, así como al hablar de la teofanía de Mambré daba su particular visión soteriológica, y en la historia de Moisés explica el valor del bautismo. Los judíos son,

29 Gregorius Ilib. De Fide 8 (98), 8-11 (Pascual Torró 1998, 146).

30 Gregorius Ilib. Tract Orig XIV 2, 8 (Pascual Torró 1997, 332).

31 Gregorius llib. De Fide (proem.) 5, 19 (Pascual Torró 1998, 54).

32 Gregorius Ilib. De Fide 7 (68), 6 (Pascual Torró 1998, 116).

33 La ortodoxia doctrinal de Gregorio de Elvira se ha pretendido poner en tela de juicio en virtud de su pertenencia al momentáneo cisma luciferiano, este es un debate en gran medida superado, véase Molina Gómez 2000, 32. 
como los cristianos, un grupo coherente de origen común, populus y plebs, pero son el populus sinagogae, y sobre la natio ludaeorum recae la consideración de plebe réproba. El pueblo de la Sinagoga ha de arrepentirse y convertirse de acuerdo con un finalismo escatológico. También los judíos procedentes de la Sinagoga entrarán a formar parte de la comunidad cristiana. La Sinagoga representa la prisca lex derogada, una realidad meramente carnal, puesto que aparece como la madre carnal incapaz de cumplir con su misión, que queda en manos del pueblo creyente, la Iglesia. La Sinagoga no es nunca más la madre de Cristo en el sentido espiritual sino madre carnal y no espiritual, pues el relevo lo ha de tomar la Ecclesia. Esta es la razón de la exégesis escenográfica de Moisés abandonado en las aguas, y que se explica no tanto por alegoría como por tipología. La Sinagoga ha terminado su misión histórica, no puede ofrecer nada más, se la compara con una mujer que se ha quedado estéril: su útero es "miserable», o es una mujer que no puede tener más hijos. La esterilidad final de la Sinagoga se debe oponer necesariamente a la fertilidad inesperada de Sara, la esposa de Abraham y prefiguración de la Iglesia. También Agar, en tanto que ancilla Sarrae, resulta ser un tipo de la Sinagoga, estando bajo la custodia de Abraham. Esto sancionaría desde el texto bíblico la subordinación de la Sinagoga, en tanto que ancilla, cuyo hijo, que representa el pueblo judío, no es sino el hijo de una esclava, el hijo de Agar, imagen de la Sinagoga, que ha nacido en la servidumbre. ${ }^{34} \mathrm{La}$ perfidia de la Sinagoga se explica ante la comisión del terrible pecado de no haber reconocido y haberlo condenado. Sobre la Sinagoga recae la acusación de miseria moral, con calificativos alusivos a su ligereza moral y su condición espiritual de adúltera, necia y su contumaz. La Sinagoga, madre de Cristo secundum carnem, es (como aparece citada en su quinto Comentario al Cantar de los Cantares), su gran enemiga, junto con los hijos de la Sinagoga, que son sinagogae populi. El viejo Israel no es sino una Jerusalén terrena, frente a la Jerusalén eterna. Frente a la nueva patria celestial de los cristianos. La vieja Jerusalén todavía se encuentra bajo la servidumbre de la ley vieja. Es cierta su anterioridad y el prestigio de su tradición, pero ya no es querida por Dios, porque no encontró allí donde reclinar su cabeza ${ }^{35}$ Ese sentido espiritual advertiría que los judíos han perdido el Reino y Cristo ha puesto sus miras en otra parte, en el nuevo pueblo elegido. La mayor antigüedad de los judíos no es la cuestión, siempre serán algo así como los hermanos mayores; por el contrario, lo que se juzga es su dignidad, y su efectividad en los nuevos tiempos cristianos, donde el viejo pueblo de Israel no parece que acabe de encontrar ni sitio ni quehacer; el pueblo judío como tal no tiene ya utilidad histórica. Su tiempo histórico ha pasado.

El alejamiento temporal de los judíos los convierte en impíos en tanto dure su rechazo de Cristo. La gens ludaeorum es calificada de incrédula y depravada. La salvación de este pueblo encuentra un obstáculo en su traición, en la perfidia judaica. La vieja ley pertenece al pasado, se hizo según criterios puramente temporales, pero ya es algo derogado. El pueblo judío ha tenido la oportunidad de creer,

34 Molina Gómez y Mateo Donet 2018.

35 La formulación de la ciudad celestial, prefigurando la sacralización del mundo civil: Gregorius Ilib. In Cant Cant V 7, 17; ibid. 8, 27 (Pascual Torró 2000, 150-152). pero aún rechaza la oferta, y permanece por el momento inflexible como incrédulo e impío. El método exegético (basado en la tipología y en la alegoría) ha proporcionado al obispo de Elvira la comprensión de los acontecimientos del Nuevo Testamento anunciados en el Antiguo, pero no solo eso; además ha encontrado en las expresiones literales un sentido trascendente alusivo a las verdades de la fe, claramente visible en la cuestión del bautismo, cuya figura son las aguas del Nilo por donde iba el niño Moisés-Cristo. Con este método también se cuestiona la legitimidad judía y se pretende completar la historia hebrea mediante su incorporación al sistema cristiano, que ofrece un programa histórico y exegético coherente, y ha tomado para sí la tradición del Antiguo Testamento, lo cual supone para los judíos tener solo una verdad parcial, que ha ignorado la verdadera fe por una mezcla de impiedad y de incapacidad para llevar a cabo la exégesis veraz de las Escrituras. ${ }^{36}$ El verdadero protagonista de la historia sagrada es el pueblo cristiano, cuya presencia en el Antiguo Testamento se rastrea a través de las innumerables tipologías. Las consecuencias de todas estas reflexiones teológicas, basadas en una determinada manera de exégesis, son la consideración del cristianismo como la forma de realización política y forma de vida querida por Dios, y considerar concretamente el judaísmo como una forma superada, que tiene que acabarse antes de la definitiva implantación del Reino de Dios. De ahí a la persecución queda poco camino que recorrer, apenas un paso que no tardará en darse. ${ }^{37}$

\section{FUENTES}

Gregorius Ilib(erritanus), In Cant(icum) Cant(icorum). 1967. Editado por Johannes Fraipont, 169-210. CCSL, 69. Turnhout: Brepols Publishers.

Gregorius Ilib(erritanus), Tract(atus) Orig(enis) de libr(is) Sanct(arum) Script(urarum). 1967. Editado por V. Bulhart, 3-146. CCSL, 69. Turnhout: Brepols Publishers.

Simonetti, Manlio. 1975. Gregorio di Elvira. La Fede. Turín: Edizioni SEI, collana Corona Patrum.

Pascual Torró, Joaquín. 1997. Gregorio de Elvira. Tratados sobre los libros de las Santas Escrituras. Madrid: Editorial Ciudad Nueva.

Pascual Torró, Joaquín. 1998. Gregorio de Elvira. Tratados sobre los libros de las Santas Escrituras. Madrid: Editorial Ciudad Nueva.

Pascual Torró, Joaquín. 2000. Gregorio de Elvira. La Fe, Madrid: Editorial Ciudad Nueva.

\section{BiBLIOGRAFÍA}

Acerbi, Silvia, Mar Marcos y Juana Torres, ed. 2016. El obispo en la Antigüedad Tardía. Madrid: Trotta editorial.

Barcala Muñoz, Andrés, 2003. "Gregorio de Elvira». En Biblioteca Antijudaica de los escritores eclesiásticos hispanos. Vol. I: siglos IV-V, Andrés Barcala Muñoz, 161-192. Madrid: Aben Ezra Ediciones.

Blumenkranz, Bernhard. 1960. Juifs et chrétiens dans le monde Occidental. París: Peeters publishers.

Buckley, Francis J. 1964. «Gregory of Elvira». New Catholic Enc VI: 3-23.

Codoñer Merino, Carmen. 1982. "Los escritores hispano-cristianos». En Historia de España, ed. Ramón Menéndez Pidal, II, 523-525. Madrid: Espasa-Calpe.

36 Simonetti 1985a, 19. Molina Gómez 2000, 73-109.

37 Noethlichs 1971; 1996; Juster 1914, 13-18; Williams 1935; Simon 1948; Seaver 1952, 51; Taylor 1961. 
Collantes, Justo. 1954. San Gregorio de Elvira. Estudio sobre su eclesiología. Granada: Facultad de Teología de Granada.

Domínguez del Val, Ursicino. 1973. «El patrimonio literario de Gregorio de Elvira». Helmántica 74: 281-357.

Dulaey, Martine 1996. «Grégoire d’Elvira pasteur: la pédagogie du prédicateur dans le sermon sur l'ospitalità de Mambré (Gén. 18)». En Vescovi e Pastori in epoca teodosiana in occasione del XVI centenario della consacrazione di S. Agostino, 396-1996. Roma: Institutum Patristicum Augustinianum.

Fernández Ardanaz, Santiago. 1983. «Gregorio de Elvira o el intento de fusión de las dos corrientes fundamentales de la primitiva antropología cristiana». En Historia de la Teología Española, ed. Andrés Martín, I, 65-72. Madrid: Fundación Universitaria Española.

García Iglesias, Luis. 1978. Los judíos en la España Antigua. Madrid: Cristiandad.

García Moreno, Luis. 1993. Los judíos de la España Antigua. Madrid: Rialp.

Juster, Jean 1914. Les juifs dans l'Empire Romain. Nueva York: Librairie Paul Geuthner.

Ladaria, Luis F. 1999. "Gregorio de Elvira y paralelismo Adán-Cristo». Gregorianum 80 (4): 677-695.

Lejay, Paul 1908. «L'heritage de Grégorie d’Elvire». Revue Benedictine XXV: 435-457.

Lomas, Francisco Javier. 1994. «Comunidades judeocristianas granadinas. Consideraciones sobre la homilética de Gregorio de Elvira». En La sociedad de la Bética. Contribuciones para su estudio, ed. Cristóbal González Román, 319-344. Granada: Universidad de Granada

Mazorra, Enrique. 1947. «El patrimonio literario de Gregorio de Elvira». Estudios Eclesiásticos 42: 387-397.

Molina Gómez, José Antonio. 2000. La exégesis como instrumento de creación cultural. El testimonio de Gregorio de Elvira. Murcia: Universidad de Murcia.

Molina Gómez, José Antonio. 2007. «San Gregorio de Elvira y el uso de la etimología bíblica durante la segunda mitad del siglo IV». Tonos Digital 13. https://www.um.es/tonosdigital/znum13/secciones/ estudios_S_elvira.htm

Molina Gómez, José Antonio y Amparo Mateo Donet. 2018. «La interpretación patrística sobre la violencia contras las mujeres y los niños. Los casos de Abraham, Sara, Agar e Ismael». Studia Ephemeridis Augustinianum 151: 75-89.

Molina Prieto, Andrés. 1998. «Claves interpretativas en la exégesis alegórica de Gregorio de Elvira». Giennium 1, 61-118.

Montserrat Torrents, José. 1989. La sinagoga cristiana. El gran conflicto religioso del siglo I. Barcelona: Muchnik Editores.
Noethlichs, Karl Leo. 1971. Die gesetzgeberischen Massnahmen der christlichen Kaiser des vierten Jahrhunderts gegen Häretiker, Heiden und Jüden. Colonia: Institut für Altertumskunde, Universität zu Köln.

Noethlichs, Karl Leo. 1996. Das Judentum und der römische Staat: Minderheiten Politik im antiken Rom. Darmstadt: WGB Academic.

Quasten, Johannes. 1941. "The Conflict of Early Christianity with the Jewish Temple Worship». Terra Santa II: 481-484.

Rengstorf, Karl Heinrich y Siegfried Kortzfleisch. 1968. Kirche und Synagoge. Handbuch zur Geschichte von Christen und Jüden. Stuttgart: Klett-Cotta.

Rodríguez Ratia, Federico. 2017. «Gregorio de Evira. Escritor, teólogo y defensor de la fe». En Autoridad y autoridades de la Iglesia antigua: Homenaje al profesor José Fernández Ubiña, ed. Francisco José Salvador Ventura, Pedro Castillo, Purificación Ubric Rabaneda y Alberto Quirota Puertas, 209-222. Granada: Universidad.

Sayas Abengoechea, Juan José. 1993. «Cuestiones controvertidas acerca de los judíos en la Historia Antigua peninsular». Espacio, Tiempo y Forma, Serie II, Historia Antigua VI: 479-528.

Seaver, James Everett. Persecution of the Jews in the Roman Empire (300-438). Kansas: University of Kansas Publications.

Simon, Marcel. 1948. Verus Israel. Étude sur les relations entre chrétiens et juifs dans l'Empire Romain (135-425). París: E. de Boccard.

Simonetti, Manlio. 1985a. Lettera e/o Allegoria. Un contributo alla storia dell'esegesi patrística. Roma: Studia Ephmerides Augustinianum.

Simonetti, Manlio. 1985b. "La tipologia di Abramo in Gregorio di Elvira». AFLFC VI: 141-143.

Suárez Fernández, Luis. 1980. Los judíos españoles en la Edad Media. Madrid: Rialp.

Taylor, R. E. 1961. «Attitudes of the Fathers toward practices of Jewish Christians». Studia Patristica IV: 504-511.

Teja, Ramón. 2005. «Exterae gentes: relaciones con paganos, judíos y herejes en los cánones de Elvira». En El concilio de Elvira y su tiempo, José Fernández Ubiña y Manuel Sotomayor, 197-228. Granada: Universidad de Granada.

Thouvenot, Raimond. 1943. «Chrétiens et juives à Grenade au IV siècle après J.C.». Hesperis XX: 206-211.

Tovar Paz, Francisco Javier. 1994. Tractatus, sermones atque homiliae: el cultivo del género literario del discurso homilético en la Hispania tardoantigua y visigoda. Cáceres: Universidad de Extremadura.

Williams, Lukyn. 1935. Adversus Iudaeos. A Bird's-Eye View of Christian Apologiae until the Renaissance. Cambridge: Cambridge University Press.

Young, Frances. 1997. Biblical exegesis and the formation of Christian Culture. Cambridge: Cambridge University Press. 